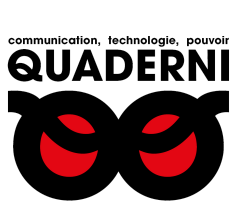

\title{
Quaderni
}

Communication, technologies, pouvoir

98 | Hiver 2018-2019

Humanités numériques : vers l'institutionnalisation

\section{Comment prévoir l'avenir métropolitain aujourd'hui ? Un problème de doctrine et de technologies numériques}

Bertrand Morvan

\section{OpenEdition}

Journals

Édition électronique

URL : https://journals.openedition.org/quaderni/1490

DOI : 10.4000 /quaderni. 1490

ISSN : 2105-2956

Éditeur

Les éditions de la Maison des sciences de l'Homme

Édition imprimée

Date de publication : 5 février 2019

Pagination : 123-134

Référence électronique

Bertrand Morvan, «Comment prévoir l'avenir métropolitain aujourd'hui ? Un problème de doctrine et de technologies numériques », Quaderni [En ligne], 98 | Hiver 2018-2019, mis en ligne le 05 février 2021, consulté le 08 janvier 2022. URL : http://journals.openedition.org/quaderni/1490 ; DOI : https:// doi.org/10.4000/quaderni. 7490 


\section{Politique}

\section{Comment}

prévoir l'avenir métropolitain aujourd'hui? Un problème de doctrine et de technologies numériques

Bertrand Morvan

Chercheur en sciences politiques, conseil en développement territorial
Ce papier veut défendre une certaine conception politique de l'urbain à partir du regard singulier d'un habitant de la banlieue parisienne. Des dispositifs techniques de validation démocratique de macro-projets techniques se mettent en place. C'est le cas de l'aménagement du Grand Paris Express, projet de réseau technique de transport en rocade entre la première couronne et la rocade routière du périphérique de Paris. Ce nouveau réseau est associé au projet métropolitain du Grand Paris. Mais c'est aussi, en proche banlieue du Val-de-Marne, dans un récent passé, la définition de plans locaux d'urbanisme engagés dans les communes de la première couronne rouge parisienne dans les années 2014-2016. Par répercussion, ces derniers sont concernés par la phase importante de métropolisation en cours de la métropole parisienne. Pourtant, se demander pourquoi des phases de concertation organisées par les édiles ne prirent pas en compte l'ampleur des enjeux environnementaux et démocratiques nous paraît pertinent comme questionnement d'avenir. L'interpellation des communes de la petite couronne de la région parisienne par l'État et son préfet de Région est le moment critique. Des contraintes décisionnelles se profilent pour les édiles avec la dynamique, très expansive, de projets métropolitains. Ces moments d'interpellation institutionnelle des pouvoirs locaux par l'instance centrale témoignent d'une relation particulière. Il s'agit d'une logique expansive de «Ville-État» sur la proche banlieue. La contrainte d'un nouveau front d'aménagement s'impose avec, en particulier, l'urgence de nouveaux programmes de création de logements sociaux. Les concepts et catégories de l'historien F. Braudel pour le cas de Paris sont à reconsidérer ${ }^{1}$. Ils n'ont pas vieilli avec le cas de la métropole parisienne. 
Il faut ajouter le rôle essentiel des grands corps techniques et des grandes entreprises aux stratégies foncières de revalorisation financière des centres-villes des communes autour de la villecentre. La prégnance du schéma symbolique fort, Paris versus banlieue, a fondé l'imaginaire politique avec l'histoire de la peur des « classes dangereuses $»^{2}$. Pourtant, certains géographes nous disent que ce schéma centre-périphérie n'est plus actuel dans la symbolique des mémoires sociales. La fondation plus ample d'une métropole moderne dépasserait ces frontières culturelles. Toute résistance symbolique et populaire serait annulée. La priorité irait à l'expansion d'une métropole trop petite avec Paris. La crédibiliser dans la course à la concurrence à l'échelle mondiale avec Londres, New-York, Tokyo, Francfort, dans la perspective tracée par S. Sassen, s'imposerait ${ }^{3}$.

Décrire, sans a priori, selon une nouvelle posture intellectuelle, ces phases de composition et de négociation à différentes échelles, s'impose à partir d' « une totalité fragmentaire et fragmentée » en pleine métamorphose $e^{4}$. Une poétique du fragment pour s'ouvrir à l'appel de l'être devient nécessaire face au " post-urbain » (F. Choay, 1992). Plus globalement, une attitude sceptique, selon la posture d'une suspension du jugement de type phénoménologique, nous paraît de bon aloi ${ }^{5}$. Des catégories de jugement naîtraient à l'écart d'une modélisation immédiate et déterministe. Les travers d'une croyance au positivisme technique, peu souple dans ses futurs effets et confinant au scientisme, peuvent être questionnés ${ }^{6}$. Les jeux politiques et stratégiques sont complexes ${ }^{7}$, qu'il s'agisse de la petite couronne à 20 kilomètres de Paris ou de la seconde couronne, aux limites de la région parisienne (à partir de 40 à 70 kilomètres).
Des résistances culturelles et donc techniques ne sont pas prises en compte alors qu'on assiste à un retour d'une planification aux orientations indicatives. De fait, de nouvelles logiques d'acteurs peuvent être identifiées lors de la délimitation géographique du Grand Paris avec l'application de la loi Métropole de décembre 2016 et précédemment, avec la loi N.O.T.R.e. d'orientation territoriale de juin 2015. La métropole parisienne doit englober trois départements avec les Hautsde-Seine, le Val-de-Marne et la Seine-Saint-Denis pour lui permettre un développement jusqu'en première couronne. Les plans locaux d'urbanisme sont concernés par la création importante de nouveaux programmes de logements sociaux et de morceaux de villes y attenant (70 000 par an en fonction de la nature du tissu urbain existant à produire). Avec 68 grandes gares, l'appropriation démocratique de logiques techniques pour l'avenir de sociétés locales est une question posée. Elle serait peu envisagée lors de « conférences publiques » qui sont des consultations ${ }^{8}$. Encore faut-il poser la question de la définition d'un processus démocratique authentique et porteur pour les « récits-images » proposés. Il s'agit de " figures au pouvoir d'évocation ${ }^{9}$, produits d'images numériques et de récits politiques d'accompagnement. Le changement technique est présenté pour l'avenir comme la voie du progrès et de la modernité.

\section{Un changement de paradigme dans la façon de définir l'avenir métropolitain}

Avec une dérive idéologique par rapport à son projet d'origine comme " praxis ", il y a une crise de l'épistémè de l'urbanisme dont le symptôme est le combat entre " anciens » et « mo- 
dernes » aménageurs ${ }^{10}$, le besoin de se prouver la scientificité des études urbaines à critiquer ${ }^{11}$ et la dérive technoscientifique vers le tout numérique associé à l'urbain des techno-réseaux. L'abandon des principes initiaux, sédimentés avec le temps, n'a pas été clairement affiché. Un désaccord des savants lors de ce changement de paradigmatique est manifeste. Par exemple, on opposera deux types d'auteurs «clés » de l'utopie, figure essentielle en urbanisme. Avec la famille des théoriciens de l'urbanisme formée au marxisme, T. Paquot, philosophe de l'urbain, disqualifiait le schéma utopique dès 1996. Mais de façon ambivalente, il le réintégrait pourtant en 2016 et en $2017^{12}$. L'autre famille est constituée de M. Abensour et P. Ricœur, philosophes discutant du rôle de l'utopie comme correctrice de l'idéologie et réciproquement à partir des thèses de l'École de Francfort ${ }^{13}$. Pour F. Choay en 1987, l'urbanisme est une pratique («praxis ») née à la fin du XIX ${ }^{\mathrm{e}}$ siècle et au début du $\mathrm{XX}^{\mathrm{e}}$ comme discipline scientifique et technique émergeante avec pour projet d'accompagner le développement industriel ${ }^{14}$. Bien que plutôt de nature artistique et pluridisciplinaire (cf. les arts urbains), elle se voulait, dés l'origine, scientifique et technique. Il fallait construire son autonomie par rapport aux autres disciplines se partageant le champ de l'intervention urbaine. Elle a souhaité se dégager des principes d'un pré-urbanisme de la Renaissance dont les fondements étaient le Traité (L.B. Alberti) et l'Utopie (T. More), puis du socialisme utopique, mais n'y ait jamais parvenu. Aujourd'hui, la dérive se traduit par la forte influence d'une association peu démocratique de groupes immobiliers et de pouvoirs publics lors de la réalisation de projets de réseaux techniques. La localisation des nouveaux pôles est le fruit d'une segmentation de l'espace à l'aide de l'outil numérique. Ces nouveaux ensembles bâtis sont envisagés souvent à distance des centresvilles pour raison d'efficacité financière et de rapidité d'exécution technique. Les géographes de l'E.U.P. disqualifiaient la construction d'un « local de proximité » sans parler de cette complexité infrastructurelle et politique ${ }^{15}$. Or une approche dite " poétique » au sens d'Aristote ${ }^{16}$ proposerait un autre regard qu'une forme systématique d'enchaînement technique des causes. Le récit à portée sensible et esthétique devient alors politique. Par exemple, les récits d'enquêtes à l'aide de photographies des flux de passants dans le centre commercial des Halles, en sous-sol et aux étages supérieurs, rappellent complètement la manière dont $\mathrm{W}$. Benjamin « travaillait » à partir d'un point de vue littéraire, dans les années 30, les traces mnésiques des passages du Paris du XIX ${ }^{\mathrm{e}}$ siècle ${ }^{17}$. Des travaux sur les « lieux métropolitains décloisonnés et transversaux et ceux qui ne le sont pas $\gg^{18}$ creusent ce sillon.

\section{Un retour inflationniste et symptomatique du plan d'aménagement régional pour la région parisienne}

Actuellement, un rapport conflictuel entre local et mondial avec le processus de métropolisation des grandes villes est le non-dit de la communication politique. L'illustration en serait le désintérêt pour l'inauguration du chantier du Grand Paris Express en son arc sud, entre Issy-les-Moulineaux et Noisy-le-Grand, en avril 2016. Une équipe d'artistes sera missionnée sur 4 années, au début de 2017 (selon le journal Le Parisien). Par ailleurs, la loi Métropole de 2016 et les dispositions législatives en janvier 2017 intègrent de 
nombreux nouveaux plans, à différentes échelles et secteurs d'intervention (climat, etc.). Pourtant, en 2009, l'ancien président N. Sarkozy critiquait le plan lors du lancement du concours d'architectes de l'Atelier International du Grand Paris intitulé : « Le Grand Pari(s) de l'agglomération parisienne » et lors de l'exposition au Palais de Chaillot. Une rigidité du droit foncier expliquait le manque d'ambition des grands projets urbains à l'échelle métropolitaine du Grand Paris ${ }^{19}$. Aujourd'hui, le préfet de Région, M. Cadot, privilégie la dynamique des appels à projets locaux. Il y a donc bien « un contexte paradoxal $»^{20}$. Les édiles, aujourd'hui, veulent se défaire de cette planification pour agir. Y aurait-il renouvellement de la tradition française avec un certain relâchement de la norme, avec l'abandon des 19 contrats de développement territoriaux de 2012 ? Certains acteurs qualifient de « prospective territoriale » les actuels plans locaux territoriaux qui sont plutôt des décisions prévisionnelles de mutations de territoires laissés en jachère ${ }^{21}$.

\section{La nécessité d'une recherche généalogique concernant les théories urbaines}

Le retour inflationniste d'une planification régionale en janvier $2017^{22}$ invite à une recherche exploratoire parmi les courants de l'urbanisme. Cela permet de déconstruire les perspectives, toujours renaissantes, d'une science dite de l'urbanologie qui a échouée ${ }^{23}$. Des sources doctrinales, peu reconsidérées ou abandonnées sans réelle justification idéologique, peuvent être une modalité d'explication. Une troisième voie serait envisageable entre les textes fondateurs de L.B. Alberti et T. More et entre « urbanisme progressiste » (Le Corbusier et ses épigones) et
" urbanisme culturaliste » (C. Sitte, architecte viennois). Comment retrouver le projet d'une doctrine autonome pour la geste urbanistique et une capacité à se déterminer dans ses objectifs face à l'omnipotence des réseaux techniques de communication, producteurs du «post-urbain » (F. Choay, 1992) ? On note, en effet, d'une part, des effets territoriaux les plus différenciés et inégaux au niveau local et d'autre part, la question du sens du projet urbain. La scientificité de l'urbanisme devrait pourtant, nous faire totalement y adhérer. Les sources socialistes, second courant d'inspiration, n'ont plus d'effet. Plus généralement, la discipline n'a jamais fait la part entre " la règle et le modèle ", soit le traité et l'utopie. On oscille alors, depuis le XIX ${ }^{\mathrm{e}}$ siècle, après le pré-urbanisme de la Renaissance, entre courants progressiste et culturaliste, jusqu'au courant technoscientifique actuel. Il n'a jamais été construit de rapport critique entre idéologie et utopie jusqu'à aujourd'hui, notamment en ce qui concerne la planification des villes ${ }^{24}$. La crise larvée de l'urbanisme planificateur métropolitain serait le reflet de son incapacité à instituer politiquement l'urbain que les acteurs, pris dans l'urgence, tentent de compenser. Le politologue A. Faure, de l'I.U.P. de Grenoble diagnostiquait le mal en novembre $2012^{25}$. Une " résistance du local $»^{26}$ témoignerait d'une non-acceptation des termes du débat tels que les aménageurs publics et privés l'imposeraient ${ }^{27}$. Les expériences espagnoles à Barcelone, aux Pays-Bas à Amsterdam et même à Londres ou Berlin sont d'un autre tonneau. Les expériences passées des métropoles canadiennes, italiennes et françaises montrent aussi toutes un échec ou un semi-échec pour faire émerger une institution productrice de vie démocratique locale pour l'ensemble 
de la communauté métropolitaine ${ }^{28}$. Selon M. Gauchet, notre rapport à l'Histoire a changé. La crise porte alors sur la possibilité de se représenter l'avenir et de le rendre intelligible ${ }^{29}$. Un contexte important d'incertitudes conduit aménageurs et planificateurs à ne plus s'entendre sur le type de représentation valide ${ }^{30}$. Les habitants et urbanistes aimeraient avoir l'idée d'une image finie de ce que sera la métropole pour en débattre. L'approche historique est alors « coincée » entre Histoire et mémoire jusqu'à atteindre " l'ère de la commémoration ». L'historien P. Nora ${ }^{31}$ entre en résonance avec le philosophe P. Ricœur ${ }^{32}$. Le mouvement dit « de la patrimonialisation de lieux de mémoires et monuments historiques " $\mathrm{s}^{\prime} \mathrm{y}$ ajoute ${ }^{33}$. Le patrimoine se trouve «pris entre l'Histoire et la mémoire » (F. Hartog, 2013). L'innovation est perçue systématiquement comme un bien. Elle s'accompagne, pour l'historien F. Hartog, d'《 un présentisme » et d'un "régime d'historicité » propre ${ }^{34}$. Lors d'un colloque sur le thème des métropoles européennes ${ }^{35}$, des géographes envisageaient le récit métropolitain comme déjà construit. Il suffirait donc de "suivre le tracé ou sillon envisagé depuis 2008 par l'ex-président Sarkozy sans rien retoucher $»^{36}$. Durant l'atelier " S'emparer du récit métropolitain ? » en mairie de Paris, un responsable de la communication de la Société du Grand Paris a monopolisé la parole et «fermé les possibles ». Pourtant, l'introduction par le géographe $\mathrm{D}$. Behar de la $9^{\mathrm{e}}$ édition des journées du Grand Paris, en janvier 2018, interrogeait une approche simpliste de la planification. Ce retour important et paradoxal n'empêcherait pas l'animation par les acteurs d'un cadre d'orientations consultatives et une coproduction sur un nécessaire temps long ${ }^{37}$.

\section{L'intérêt d'une posture sceptique face aux nou- veaux développements métropolitains}

Nombre de situations de concertation locale ne changent pas fondamentalement la règle du jeu démocratique. Sont-elles les plus adaptées pour créer l'avenir de manière innovante? Avec les plans locaux d'urbanisme à valider, nous n'aurions plus à faire qu'à des « boîtes noires $\|^{38}$ lors de densifications urbaines de centres-villes. L'habitant de la banlieue Est de première couronne, interpellé lors de concertations pour la définition des plans locaux d'urbanisme, n'en peut mais si on lui demande son avis sur des propositions produites à l'avance, sans vraie vulgarisation des enjeux. Le cabinet d'urbanisme et le projet de ville municipale ne permettent pas de se projeter dans l'avenir selon des scénarii contrastés. Il s'agirait alors d'une prise de risque dans « une société du risque » (U. Beck, 2001). Agir en citoyen et /ou décideur deviendrait complexe alors qu'on n'a pas réfléchi aux déterminants d' ' une raison pratique ", particulièrement pour l'édile, (V. Descombes, 2011). À l'horizon, des valeurs technocratiques soutiennent un récit fermé à «faire passer $»^{39}$. Le décideur ne peut plus s'appuyer sur l'autorité indiscutable d'une tradition fondée sur un ensemble stocké d'enseignements et d'expériences pour décider de l'avenir dans le monde hypermoderne et $«$ liquide $»^{40}$. Par précaution, certaines expériences techniques et scientifiques peuvent se révéler préjudiciables à l'environnement et aux équilibres humains et planétaires actuels ${ }^{41}$. S'y ajouteraient l'accélération du temps numérique et l'expression d'une volonté de toute puissance. On assisterait à « la liquidation du futur $»^{42}$. Or l'ouverture maximum des « possibles » permettrait d'éviter une instru- 
mentation. Cette normativité produite d'en haut ne provoquerait pas une adhésion ${ }^{43}$. La rigidité, à long terme, du schéma d'implantation géographique du Grand Paris Express et les problèmes de mobilité en seconde couronne en seraient les conséquences avec le retour, en 2016, de combats politiques engendrés par de nouveaux redécoupages territoriaux intercommunaux.

\section{La place du numérique dans la pratique ur- banistique et ses limites pour une prospective}

Les derniers développements doivent beaucoup à la numérisation de l' " acte de bâtir » (F. Choay, 1980). Selon Laurent Devisme, professeur d'urbanologie à l'E.N.S.A.E. de Nantes: "Que l'urbanisme soit intrinsèquement lié à la production d'images iconiques et verbales tend désormais à être bien reconnu, au-delà de l'iconoclasme qui a cristallisé une fraction de la pensée critique. Une approche pragmatique des activités urbanistiques, telle qu'Ola Söderström (2001) a pu la pratiquer, peut ainsi rappeler que " l'image cristallise une fiction, un monde spatial vraisemblable au sein duquel on peut agir » (M. Lussault 2007: 57). Il reste cependant une indétermination relative au collectif d'action ici convoqué (le «on»). Qui peut donc agir et selon quelles modalités, au-delà des producteurs de figures que sont les architectes et urbanistes (...) ? D'ordre technico-politique, l'urbanisme relève notamment de productions cognitives liées à l'anticipation et la vision. Il s'agit bien d'orienter, dans le temps et l'espace, l'urbanisation, la répartition des objets spatiaux, de proposer un partage et une articulation entre espaces publics et privés, entre fonctions urbaines. À ces fins, on peut s'intéresser à tout un ensemble de techno- logies d'aide à la décision parmi lesquelles des modèles, des chartes, guides, mais aussi des images iconiques et verbales (Chapel, 2010). Le nombre de visualisations urbanistiques augmente, tantôt produites au sein de la maîtrise d'ouvrage cherchant à stabiliser son fonctionnement sur l'ensemble d'un territoire, tantôt produites en externe, par des architectes, urbanistes, consultants incarnant plutôt des démarches spécifiques sinon singulières. Le déploiement du numérique a permis une véritable extension de ces images dont plusieurs ensembliers du bâtiment, grands acteurs privés de la production urbaine, sont particulièrement friands, sous la bannière des «smart cities». Dans ce contexte, les figures peuvent être entendues comme des embrayeurs de l'action de projet; elles sont supposées engager vers un monde à construire, donner envie tout en crédibilisant la possibilité de la transformation spatiale $(. ..) »^{44}$. Une forte normativité se présente sur un mode descriptif lors de conférences municipales. De fait, on peut analyser « la carrière » de ces figures stockées dans des bases de données. L'instabilité des références n'empêche pas la « cross fertilisation » et le décloisonnement des échanges pour différents corps de métiers. Encore faut-il « pratiquer le contrôle avec le critère de traçabilité, pour garder le meilleur à l'aide du scalpel de l'analyste, particulièrement en ce qui concerne la production des bases de données $\gg{ }^{45}$.

\section{Des pistes pour une prospective critique}

Qu'est-ce qui a changé, depuis le S.D.A.U. de 1965 de P. Delouvrier, grand commis de l'État, chargé de l'aménagement de la région parisienne jusqu'au projet du Grand Paris de 2008 ? Les images à l'époque n'étaient pas contradictoires 
avec la manière dont le public se les appropriait. Leur traduction se faisait selon des prismes populaires d'interprétation avec des transformations déviantes ${ }^{46}$. Aujourd'hui C. Favier, président du conseil général du Val-de-Marne, organise une défense de l'identité territoriale contre le projet d'élimination du département à l'aide d'une réinterprétation de cette histoire. Il serait, avec la Seine-Saint-Denis, l'Essonne, englobé par la Métropole. L'archivage de la mémoire « à la Raymond Depardon »s'appuie sur des photographies de paysages depuis sa naissance en 1969. Des fictions hétérotopiques, en marge de la programmation et des discours officiels des grands aménageurs, sont appelées ${ }^{47}$. Finalement, le diagnostic d'une crise de l'épistémè de l'urbanisme permettrait l'ouverture à la prospective des « nouveaux-nouveaux mondes » (G. Balandier, 1993). L'analyse des modalités d'appropriation démocratique des nouvelles images virtuelles nous invite à distinguer l'étude de la nature des supports imagiers utilisés de celle des modalités contextuels des cadres cognitifs orientant l'action lors de leur utilisation. Mais les récits-images de grands décideurs régionaux du conseil de ParisMétropole sont un puissant obstacle épistémologique à la connaissance objective des territoires. A contrario, une saisie poétique des images dialectiques et du récit fragmentaire éviterait de « passer à côté » des métamorphoses contradictoires et non-résolues. Plus généralement, aborder les enjeux d'une « praxis » d'intervention sur l'avenir pour un édile ou tout autre intervenant du conseil est pertinent ${ }^{48}$. Des pistes exploratoires et complémentaires d'une prospective « contrefactuelle » ouvrent les possibles de scénarii nouveaux ${ }^{49}$.

\section{Conclusion}

L'histoire des idées des théories urbaines étaye la critique du fort tropisme modélisateur et simplificateur de propos d'urbanistes-aménageurs du Grand Paris. Partant de projets de communes de banlieue parisienne, nous avons dégagé des catégories critiques. Des contradictions portées par les discours politico-techniques et les inégalités socio-spatiales laissées ou produites après coup seraient identifiables. Pourtant, en amont de macro-systèmes techniques, un déterminisme sidérant est porté par l'actuel récit métropolitain, comme la communication de la Société du Grand Paris l'illustrait lors d'un colloque international de 2016 en Mairie de Paris. Une simple description de ce qui existe déjà comme contextes locaux, avant toute modélisation numérique, aurait permis d'éviter quelques erreurs épistémologiques. Il n'y a donc pas vraiment de solution démocratique donnée à "l'économie $d$ 'archipel $\aleph^{50}$. Des solutions techniques, " gravées dans le marbre » en ce qui concerne l'interconnexion territoriale du Grand Paris Express, le confirmeraient. Des communes « qui gagneront et d'autres qui perdront » est l'avenir post-urbain proposé pour un espace périurbain ${ }^{51}$. Tenir compte du phénomène d'une croyance nécessaire accompagnant l'action selon une métaphysique de la connaissance et faire alors un détour épistémologique pour revenir à une connaissance plus universelle sont les priorités. Son partage construirait, dans l'échange sans frontière, un sens commun dans la Cité ${ }^{2}$. 


\section{$R \cdot \dot{E} \cdot F \cdot \dot{E} \cdot R \cdot E \cdot N \cdot C \cdot E \cdot S$}

K. AXELOS, Ce qui advient, Encre marine, Paris, 2009.

ARISTOTE, De la poétique, Mille et une nuits, Paris, 2006.

F. CHOAY, La règle et la méthode. Sur la théorie de l'architecture et de l'urbanisme, Seuil, Paris, 1980. Anthropologie de l'espace, Seuil, Paris, 1992.

ESPRIT, L'avenir du pouvoir local, Paris, 02/2015.

G. BALANDIER, Le Grand Dérangement, Paris, PUF, 2005.

F. HARTOG, Croire en l'histoire, Flammarion, Paris, 2013.

K. MANNHEIM, Idéologie et Utopie (1929), Rivière, 1956.

T. PAQUOT, L'utopie ou l'idéal piégé, Essais Poche, Paris, 1996. Lettres à Thomas More sur son utopie (et celles qui nous manquent), La Découverte, 2016. Dicorue. Vocabulaire ordinaire et extraordinaire des lieux urbains, C.N.R.S.-éd., Paris, 2017

B. MORVAN, « Émergence d'un récit contradictoire du périurbain dans la métropole du Grand Paris: le cas de l'Institut du Mont Ezard à Villecresnes au sud-est du Val-de-Marne », Quaderni n $^{\circ} 86,1 / 2015$ et « Un récit métropolitain en constitution au Perreux-sur-Marne », Quaderni n89, 01/2016

O. MONGIN, La Ville des flux : L'envers et l'endroit de la mondialisation urbaine, Fayard, 2013 J. RANCIÈRE, Les bords de la fiction, Seuil, Paris, 2017.

P. RICEUR, Temps et récit, Seuil, 1983-1991 et La mémoire, l'histoire, l'oubli, Seuil, Paris, 2000.
C. TOPALOV, «Trente ans de sociologie urbaine. Un point de vue français ", Metropolitiques, 16.10.2013.

C. TIERCELIN, Le doute en question : parades pragmatistes au défi sceptique, Paris, Éditions de l'éclat, 2005.

P. VELTZ, Mondialisation, villes et territoires. L'économie d'archipel, Seuil, Paris, 1996 
$\mathrm{N} \cdot \mathrm{O} \cdot \mathrm{T} \cdot \mathrm{E} \cdot \mathrm{S}$

1. «Un petit Paris dans le Grand Paris?». Intervention du philosophe $\mathrm{O}$. Mongin, in « Du Grain à moudre », France Culture, 14.12.2017.

2. L. Chevalier, Classes laborieuses, classes dangereuses, réd. 2002, Perrin, Paris.

3. S. Sassen, The global city, New York, London, Tokyo. Princeton, Princeton University Press, 1991 et les propos de M. Vanier et D. Behar dans le cadre d'un entretien critiquant un pouvoir local fait de proximités entretenues, Esprit, « L'avenir du pouvoir local », 02/2015.

4. K. Axelos, Ce qui advient, Encre marine, Paris, 2009 et En quête du sens, Encre marine, Paris, 2010.

5. J.L. Marion, Reprise du donné, PUF, Paris, juin 2016.

6. Cf. la critique de la modélisation arrêtée du futur schéma de Grand Paris Express lors de la conférence générale de G. Dupuy en mars 2013 à l'I.U.P. : « Retour sur l'urbanisme des réseaux "».

7. B. Morvan, «Émergence d'un récit contradictoire du périurbain dans la métropole du Grand Paris: le cas de l'Institut du Mont Ezard à Villecresnes au sud-est du Val-de-Marne », Quaderni, 01/2015, n86.

8. B. Morvan, «Un récit métropolitain en cours de constitution au Perreux-sur-Marne ", Quaderni, 01/2016, no89.

9. L. Devisme, «Figures urbanistiques en régime prospectif. Pour une critique des pouvoirs d'évocation ", Journal of Urban Research, special issus 7, 2015.

10. $8^{\mathrm{e}}$ Journées Grand Paris : «Peut-on mettre le Grand Paris en plans? », E.U.P., Marne-La-Vallée, 19 et 20 01.2017. Le deuxième jour, « anciens » et « modernes » s'opposaient sur les problèmes de représentation des enjeux territoriaux en banlieue nord-est de Paris.

11. C. Topalov, « Trente ans d'histoire de la sociologie urbaine française. Un point de vue français » Metropolitiques, 1993.

12. T. Paquot, Lettres à Thomas More sur son utopie (et celles qui nous manquent), La Découverte, 2016.

13. M. Abensour, Pour une philosophie politique critique, Sens et Tonka, 2009.

14. F. Choay, «Urbanisme », in Encyclopedia Universalis, 1987.

15. « L'avenir du pouvoir local », in Esprit, Paris, $02 / 2015$

16. Aristote, De la poétique, 335 après J.C. Rhétorique, art dramatique et poésie y étaient fondés.

17. A. Jarrigeon, « Beaubourg, les Halles, la Villette : comment trouver sa place? », in Esprit 2012/11 et colloque « Médias et Métropole parisienne », Mairie de Paris, 17.09.2013.

18. Atelier «La métropole dans le local: les lieux métropolitains », Cabinet Ville Ouverte, Journées Grand Paris, École d'urbanisme de Paris 19 et 20 mars 2015. 19. Discours d'inauguration de N. Sarkozy pour l'exposition de l'Atelier International des Architectes pour le projet du Grand Paris. Cité de l'architecture et du patrimoine. Palais de Chaillot, 29.04.2009.

20. Introduction de D. Behar, «Peut-on mettre le Grand Paris en plans? », op. cité.

21. Cf. la présentation du plan local de l'habitat intercommunal de Saint-Quentin-en-Yvelines par une directrice de « la prospective et de la planification » de la communauté d'agglomération le 19.1.2017 à l'E.U.P.: « Peut-on mettre le Grand Paris en plans? », op. cité. 22. Cf. introduction de D. Behar au séminaire « Peut-on mettre le Grand Paris en plans? » de l'E.U.P. op. cité. 23. Cf. l'introduction de F. Choay intitulée « l'urbanisme en question » dans le livre L'urbanisme, utopies et réalités, Seuil,1965

24. F. Choay, La règle et la méthode. Théorie de l'architecture et de l'urbanisme, Seuil, 1980.

25. A. Faure, «Les métropoles à l'épreuve de leur récit 
politique » in colloque « Governing the Metropolis: powers and territories. New directions for research"L.A.T.T.S.- C.N.R.S., Noisy-le-Grand, 2012.

26. Introduction de D. Behar aux journées Grand Paris, « Le Grand Paris et la question métropolitaine - Controverses, stratégies et projets ", Institut d'urbanisme de Paris, Créteil, 21 et 22. 03. 2011.

27. Colloque "Gouvernance et stratégies des métropoles européennes » en Mairie de Paris des 23 et 24.05.2016.

28. A. Faure, «Les métropoles à l'épreuve de leur récit politique » dans le colloque, op. cité.

29. M. Gauchet, La condition politique, Gallimard, 2005.

30. Atelier de la journée du 20.01.2017 «Peut-on mettre le Grand Paris en plans? » op.cité. Pour un « ancien » aménageur de Plaine Commune dans les années 90, qu'oublie-t-on lors de la représentation des enjeux stratégiques autour des centres directionnels avec les cartes numérisées par rapport aux cartes dessinées « au fil du crayon » et commentées de façon dynamique ? 31. P. Nora, Lieux de mémoire; Les Frances, III, 1992 et Présent, nation, mémoire, Gallimard, 2011.

32. P. Ricœur, La mémoire, l'histoire, l'oubli, Seuil, Paris, 2000.

33. Max Querrien, Pour une nouvelle politique $d u$ patrimoine, Documentation française, 1982

34. La Grande Table-France Culture, "Que peut-on encore prédire? », 2.01.2017 avec F. Hartog, historien et J. Attali, économiste.

35. Colloque international «Gouvernance et stratégies métropolitaines », Mairie de Paris des 23.05.et 24.05.2016.

36. Conclusion du géographe M. Vanier le 24.05.2016 en Mairie de Paris. Colloque « Gouvernance et stratégies métropolitaines » op.cité.

37. D. Behar, «Aménagement du territoire : le temps long de la planification a-t-il encore un avenir ? » sur le site de 1'U.P.E.C.- Paris- Est, 7.12.2017.

38. B. Morvan, « Un récit métropolitain en constitution au Perreux-sur-Marne », Quaderni nº89, 01/2016 op.cité.

39. Intervention du représentant de la société du Grand Paris lors de l'atelier " S'emparer du récit métropolitain ? ». Colloque "Gouvernance et stratégies métropolitaines » en Mairie de Paris op. cité.

40. Z. Baumann, Le présent liquide, Seuil, Paris, 2007. 41. H. Jonas, Le principe responsabilité, Vrin, Paris, 1979 .

42. Hervé Juvin, « La crise d'un monde fini ? » Le Débat, $\mathrm{n}^{\circ} 155,2009$, p. 4-22.

43. A. Faure, «Les métropoles à l'épreuve de leur récit politique : Le transcodage contrarié des émotions et de la technique ». Colloque de 2012 du L.A.T.T.S. op. cité. 44. L. Devisme, "Figures urbanistiques en régime prospectif. Pour une critique des pouvoirs d'évocation ", Journal of Urban Research, special issus 7, 2015.

45. P.M. Menger, sociologue, dans « La suite dans les idées », France Culture, 11.112017.

46. C. Orillard, «Entre la Documentation Française et Paris-Match, la communication paradoxale du schéma directeur de 1965 ». P. Delouvier, en 1965, n’hésitait pas à faire campagne auprès du public à l'aide d'articles et d'images dans l'hebdomadaire Paris-Match risquant la trahison effective des journalistes.

47. J. Rancière, Les bords de la fiction, Seuil, 2017. L'intérêt sera porté sur « les bords de la fiction » où le temps du récit peut se construire de façon hétérogène par rapport au temps des décideurs, lors de « microévènements » comme les récits d'échecs des villes nouvelles Val-de-Reuil en Normandie ou de l'Isle d'Abau, en banlieue de Lyon, créée en 1968.

48. Séminaire «Peut-on mettre le Grand Paris en plans ? » de l'E.U.P le 20.01.2017 op.cité. M ${ }^{\text {me }}$ Elsa Sacksick, du cabinet Adden, évoquait la coproduction 
avec la société civile, du contentieux de la planification environnementale.

49. Quentin Deluermoz et Pierre Singaravélou, «What If...? Apports, limites et enjeux de la démarche contrefactuelle en histoire » [archive], E.H.E.S.S., 2010. 50. P. Veltz, Mondialisation, villes et territoires. L'économie d'archipel, Seuil, 1996.

51. Voir les propos critiques G. Dupuy en 2013 sur la rigidité des modèles utilisés en comparaison de ceux des fondateurs de l'urbanisme des réseaux. Conférence générale « Retour vingt ans après sur le livre : L'urbanisme des réseaux-Théories et méthodes » (Grand Paris, I.U.P.-Créteil, Janvier 2013). Marc Wiel, urbaniste, notait aussi l'insuffisance de « forces de frappe » de l'aménagement des communes du périurbain, Premières Assises du Tiers - espace et du périurbain, 14 novembre 2013, Villecresnes.

52. C. Tiercelin, Le doute en question : parades pragmatistes au défi sceptique, Paris, Éditions de l'éclat, 2005 et Les chemins de la philosophie, France Culture, 15.11.2017.

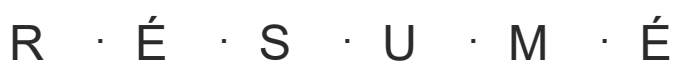

L'histoire des idées des théories urbaines étaye la critique de propos d'aménageurs du Grand Paris beaucoup trop simplificateurs. Partant de projets de communes de banlieue parisienne, nous avons dégagé des catégories critiques. Nous avons pu identifier des contradictions portées par les discours politicotechniques et les inégalités socio-spatiales laissées ou produites après coup. Pourtant, en amont de projets $\mathrm{du}$ type macro-systèmes techniques de transport (A. Gras, 1997), un très fort déterminisme apparaît dans l'actuel récit métropolitain. À ses débuts, la communication de la Société du Grand Paris l'illustrait. Une simple description de ce qui existe déjà comme contextes locaux, avant toute modélisation numérique, aurait permis d'éviter quelques erreurs épistémologiques. Il n'y a donc pas vraiment de solution démocratique donnée à « l'économie d'archipel » (P. Veltz, 1996). Tout un espace de la seconde couronne n'est pas concerné par le projet d'interconnexion territoriale du Grand Paris Express (G. Dupuy, 2013 et M. Wiel, 14.11. 2013). La croyance (et son récit) accompagnant l'action appelle à faire un détour épistémologique pour revenir à une connaissance plus universelle. Des ateliers d'urbanisme ouverts à tous, aideraient à construire un sens commun dans la Cité (C. Tiercelin, 2005).

\footnotetext{
Abstract

The history of urban theories supports the criticism of the words of the organizers of Greater Paris, which are far too simplistic. Starting from projects in the suburbs of Paris, we have identified critical categories. We have been able to identify contradictions brought by politicotechnical discourses and socio-spatial inequalities left or produced afterwards. Yet upstream of projects of the type "macro-technical transport systems" (A. Gras,
} 
1997), a very strong determinism appears in the current metropolitan narrative. At the beginning, the communication of the Société du Grand Paris illustrated it. A simple description of what already exists as local contexts, before any numerical modeling, would have made it possible to avoid epistemological errors. So there is no real democratic solution to the "archipelago economy" (P. Veltz, 1996). A whole area of the second ring is not concerned by the Grand Paris Express territorial interconnection project (G.Dupuy and M.Wiel, 2013). The belief (and its narrative) accompanying the action calls for an epistemological detour to return to "a more universal knowledge" (C. Tiercelin, 2015). Urban planning workshops open to all, would help to build common sense in the City. 